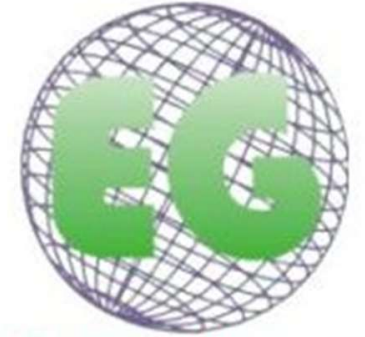

ISSN 1695-6141 N58

\title{
Orientaciones formativas para un cuidado humanizado en enfermería: una revisión integrativa de la literatura
}

Training guidelines for humanised nursing care: an integrative literature review

\author{
Mercedes Díaz-Rodríguez ${ }^{1}$ \\ Lucía Alcántara Rubio ${ }^{1}$ \\ David Aguilar García ${ }^{1}$ \\ Esther Puertas Cristóbal ${ }^{1}$ \\ Mercedes Cano Valera ${ }^{1}$
}

1 INDESS (Instituto Universitario de Investigación para el Desarrollo Social y Sostenible), Universidad de Cádiz. Cádiz. España. mercedes.diaz@uca.es

\section{https://doi.org/10.6018/eglobal.392321}

Recibido: 2/08/2019

Aceptado: 18/09/2019

\section{RESUMEN:}

Introducción: La comunicación y la relación interpersonal son los elementos más esenciales para un cuidado humanizado. El establecimiento de una relación más allá de la atención física a través de la humanización de las acciones en el plan de cuidados, favorece el proceso de recuperación; sin embargo, tanto en la formación como en la práctica enfermera se mantiene la inercia de un enfoque técnico y neopositivista, dejando de lado tales aspectos.

Objetivo: Aportar conocimientos más específicos sobre la importancia de situar y orientar los cuidados de la enfermería desde una proyección biopsicosocial, con un enfoque humanizado, poniendo especial énfasis en las competencias comunicativas y relaciones interpersonales como elementos esenciales.

Metodología: Revisión bibliográfica integrativa, con análisis crítico de la literatura consultada, que incluye artículos originales y de revisión publicados en castellano, portugués e inglés de 2013 a 2018. Para la búsqueda se han consultado las bases de datos SciELO, MEDLINE/Pubmed, Web of Science y CUIDEN.

Resultados: Se han extraído cuatro categorías de análisis que dan como resultado 1) las habilidades comunicativas y emocionales como elementos esenciales del cuidado humanizado, 2) la necesaria capacitación emocional y comunicativa en la formación de enfermería, 3) otros factores que contribuyen a un cuidado humanizado y 4) cambios metodológicos y recursos pedagógicos para la capacitación comunicativa y emocional de docentes, alumnado y profesionales de enfermería.

Conclusiones: Se reclama la necesidad de incorporar, de manera más pedagógica y profunda, programas formativos en competencias emocionales y de comunicación en enfermería para un cuidado humanizado.

Palabras clave: Educación en enfermería, Humanización de la atención, Atención en enfermería, Comunicación, Relaciones Enfermero-Paciente. 


\begin{abstract}
:
Introduction: Communication and interpersonal relationships are the most essential elements of humanised care. The process of recovery is fostered by establishing relationships outside of physical care through the humanisation of actions in the care plan. However, in both nursing training and nursing practice, the inertia of a technical and neo-positivist approach persists and such aspects are therefore neglected.

Objective: To provide more specific knowledge on the importance of considering nursing care from a biopsychosocial perspective, with a humanised approach, placing special emphasis on communicative skills and interpersonal relationships as essential elements.

Methods: An integrative literature review, with a critical analysis of the literature consulted, including original articles and reviews published in Spanish, Portuguese, and English from 2013 to 2018. The SciELO, MEDLINE/PubMed, Web of Science, and CUIDEN databases were searched.

Results: The following four categories emerged from this analysis: 1) communicative and emotional skills as essential elements of humanised care; 2) the necessary emotional and communicative training in nursing education; 3) other factors contributing to humanised care; and 4) methodological changes and pedagogical resources for the communicative and emotional training of lecturers, students, and nursing professionals.

Conclusions: There is a need to develop, in a more pedagogical and profound way, training programmes in nursing on emotional skills and communication for humanised care.
\end{abstract}

Key words: Nursing Education, Humanisation of Assistance, Nursing Care, Communication, NursePatient Relations.

\title{
INTRODUCCIÓN
}

El acto de cuidar, actualmente, está siendo deficiente, tanto en la práctica profesional sanitaria como en la formación. Las instituciones sanitarias se mantienen en un enfoque del modelo biomédico, desde el que se realizan acciones de cuidado centradas en el procedimiento o en la técnica y no en la persona y en su integridad. Este enfoque, sustentado en el paradigma positivista de la categorización, ha provocado la deshumanización de la atención, reduciendo lo humano a lo biológico y la atención hacia la curación de la enfermedad, alejando el cuidado enfermero de su visión y misión humanista y holística.

En este sentido, ante el incremento de deshumanización en salud, se hace necesario rescatar el aspecto humano por parte de los profesionales de enfermería, en tanto que es el profesional quien tiene la responsabilidad, en última instancia, de decidir de forma personal, el modo y tipo de relación que se va a establecer entre él, el paciente y la familia.

De este modo, reflexionar y prestar atención sobre la forma que interactuamos y nos relacionamos con las personas necesitadas de cuidados desde un enfoque humanizado, es especialmente importante cuando se trata de la profesión enfermera.

Existe consenso en la literatura en situar la comunicación como instrumento y herramienta mediadora de la humanización de los cuidados ${ }^{(1)}$ Una comunicación eficaz debe ser utilizada como parte del trabajo enfermero; el desarrollo de habilidades comunicativas es esencialmente importante para garantizar eficiencia en los servicios de salud; debe ser uno de los ejes centrales a los que prestar especial atención, en tanto que es a través de ésta, desde la que lograr una relación interpersonal efectiva, que posibilite la confianza mutua, la expresión de emociones, el sentimiento de tranquilidad y de bienestar. Por consiguiente, el fenómeno cuidar se revela a través de un proceso comunicativo e interactivo(2). Asimismo, se destaca que el establecimiento de una relación más allá de la atención física a través de la humanización de las acciones desarrolladas, favorece el proceso de recuperación ${ }^{(3)}$. 
Sin embargo, en la bibliografía objeto de esta revisión, se señala, por un lado, la escasa capacitación en competencias comunicativas y emocionales que los propios profesionales de enfermería reconocen que poseen; por otro lado, se apunta la escasa presencia de espacios de aprendizaje desde los que se capacite en estas competencias dentro de la formación de grado y de posgrado de las enfermeras ${ }^{(4)}$; y en caso de haberlos se suelen llevar a cabo desde un enfoque expositivo que poco ayuda al desarrollo de tales competencias.

Realizar cambios y mejoras en el tratamiento y formación de las habilidades de comunicación y emocionales, constituye una necesidad que debe ser priorizada, tanto en el ámbito académico, tomando el suficiente espacio curricular y proporcionando cambios metodológicos que permitan aprendizajes experienciales, como durante toda la carrera profesional de posgrado, al ser elementos vehiculares para alcanzar la calidad de la atención. Mejorar la capacitación de los profesionales de enfermería en estas habilidades, mediante la incorporación de nuevas metodologías y recursos didácticos que contribuyan a un aprendizaje significativo, tendrá beneficios directos en la humanización de los cuidados y en un mejor desempeño profesional de la enfermería, así como la mejora en la satisfacción y recuperación de las personas necesitadas de cuidados en los servicios de salud.

De ahí que consideremos que el presente estudio es pertinente, ya que, a partir de un análisis integrativo de la literatura al respecto, tratamos de aportar conocimientos más específicos sobre la importancia de situar y/u orientar los cuidados de la enfermería desde una proyección biopsicosocial, proporcionando especial atención a la competencia emocional y comunicativa desde un enfoque de cuidado humano integral.

De modo que los objetivos que nos proponemos son los siguientes:

- Fundamentar la importancia del desarrollo de la competencia comunicativa y emocional para el establecimiento de una relación interpersonal significativa para un cuidado humanizado en la profesión de la enfermería.

- Reflexionar sobre la necesidad de mejorar la formación del grado de enfermería de cara a favorecer la competencia comunicativa y emocional en los futuros profesionales de la enfermería.

- Visibilizar cambios metodológicos y estrategias pedagógicas que se proponen para mejorar la formación y capacitación en competencias comunicativas y emocionales en estudiantes de enfermería.

\section{METODOLOGÍA}

El trabajo que se presenta se centra en una revisión integrativa que, desde un enfoque crítico y reflexivo, pretende recoger la información más relevante de la bibliografía científica sobre la importancia de la humanización de los cuidados y la necesidad de desarrollar la competencia comunicacional y emocional en los profesionales de enfermería. Para ello, se ha realizado una búsqueda bibliográfica basada en referencias publicadas en tres bases de datos: SciELO, MEDLINE/Pubmed, CUIDEN. La búsqueda se ha llevado a cabo en el primer 
semestre de 2019. Los descriptores que hemos empleado han sido: "Educación en enfermería", "Humanización de la atención", "Comunicación" y "Relaciones Enfermero-Paciente". Para asegurar el uso de descriptores en lenguaje controlado se utilizaron las plataformas DeCS y MeSH.

La trayectoria metodológica de este estudio se basó en los siguientes pasos: lectura exploratoria (afinidad del objeto de estudio en título y resumen), selectiva, analítica e interpretativa. Se han incluido artículos originales y de revisión publicados en castellano, portugués e inglés de 2013 a 2018. Han quedado excluidos trabajos de tesis, disertaciones, boletines epidemiológicos, libros, y los artículos que no se ajustaban al periodo establecido o que no guardaban relación directa con nuestros objetivos.

Se encontraron inicialmente un total de 788 documentos. Después de la eliminación de los duplicados y tras la lectura exploratoria, se seleccionaron 308 publicaciones. Tras la lectura selectiva fueron eliminados 196, siendo la muestra final de 29 artículos. A través de la lectura crítica y un análisis integrador de todos ellos agrupados por áreas temáticas, se identificaron cuatro categorías centrales.

Figura 1: Diagrama de flujo

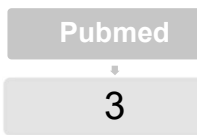

3

SciELO

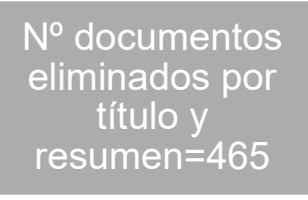

$\mathrm{N}^{\circ}$ documentos

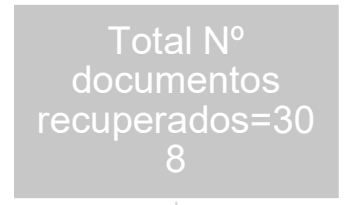

17

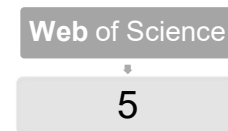

\section{Cuiden}

4

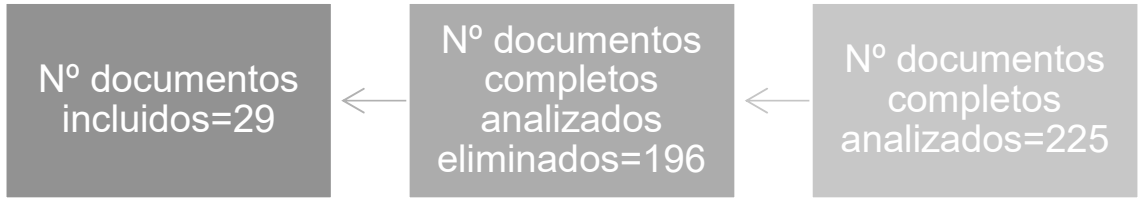

Fuente: elaboración propia

\section{RESULTADOS}

A continuación, se presenta una tabla con los resultados destacados de los 29 artículos seleccionados. 


\begin{tabular}{|c|c|c|}
\hline Autores & Metodología & Resultados \\
\hline $\begin{array}{l}\text { Mastrapa } Y \\
\text { Lamadrid } M \\
\text { (2016) }\end{array}$ & $\begin{array}{l}\text { Revisión } \\
\text { Bibliográfica } \\
\text { Integrativa }\end{array}$ & $\begin{array}{l}\text { El profesional de enfermería en las diferentes situaciones de enfermería se } \\
\text { relaciona con pacientes y familiares, fundamentalmente a través de la } \\
\text { comunicación, donde aplica diferentes elementos. Esto favorece que la } \\
\text { enfermera realice un plan de cuidado personalizado mediante una relación } \\
\text { recíproca. Una buena relación enfermera-paciente contribuye con el } \\
\text { mantenimiento de la salud, la propuesta de plan de cuidados y mejora la } \\
\text { actividad de enfermería. }\end{array}$ \\
\hline $\begin{array}{l}\text { Cestari V, } \\
\text { Moreira T, } \\
\text { Pessoa V, et } \\
\text { al. (2017) }\end{array}$ & co- & $\begin{array}{l}\text { El cuidado es un proceso interactivo que se desarrolla en la relación con el } \\
\text { otro. Respetar la integridad del ser en su deber ser debe tenerse como prioridad } \\
\text { en el cuidado de la enfermería, mediante comportamientos que lo prioricen. }\end{array}$ \\
\hline $\begin{array}{l}\text { Pott FS, } \\
\text { Stahlhoefer } \\
\text { T, Felix JVC, } \\
\text { Meier MJ. } \\
\text { (2013) }\end{array}$ & tivo, & $\begin{array}{l}\text { El establecimiento de la comunicación estuvo presente en el } 37,5 \% \text { de los } \\
\text { procedimientos realizados por el equipo multiprofesional, el aspecto humano de } \\
\text { la atención no se tiene en cuenta durante la atención brindada al individuo, que } \\
\text { puede estar relacionada con las altas tecnologías que impregnan los entornos } \\
\text { críticos. La distancia entre el equipo, el paciente y la familia compromete la } \\
\text { calidad de la atención. Se establece la comunicación como un instrumento } \\
\text { mediador de la humanización de la atención. El establecimiento de una relación } \\
\text { más allá del cuidado físico, a través de la humanización de las acciones } \\
\text { desarrolladas, favorece un proceso de recuperación de calidad }\end{array}$ \\
\hline $\begin{array}{l}\text { Moreno, I., } \\
\text { Siles, J. } \\
\text { (2014) }\end{array}$ & 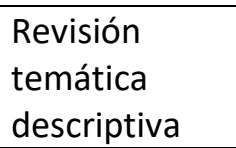 & $\begin{array}{l}\text { La práctica reflexiva y el pensamiento crítico se muestran necesarios para } \\
\text { entender la enfermería sociocrítica. }\end{array}$ \\
\hline $\begin{array}{l}\text { Michelan VC } \\
\text { de A, Spiri } \\
\text { WC (2018) }\end{array}$ & & $\begin{array}{l}\text { La humanización implica la atención integral del paciente, relacionándola con el } \\
\text { contexto familiar y social. El profesional de enfermería debe repensar sus } \\
\text { acciones, para garantizar la dignidad del ser humano en otros aspectos que van } \\
\text { más allá de la técnica, así como del entorno laboral, en su subjetividad y en los } \\
\text { aspectos culturales. }\end{array}$ \\
\hline $\begin{array}{l}\text { Uno } M \text {, } \\
\text { Tsujimoto } T \text {, } \\
\text { Inoue } T \text {. } \\
\text { (2017) }\end{array}$ & 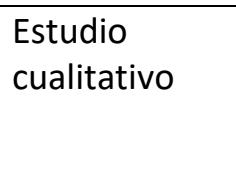 & $\begin{array}{l}\text { Eia comunicativa, Comprensión empática, Escuchando, Trato } \\
\text { les y explicaciones confiables }\end{array}$ \\
\hline $\begin{array}{l}\text { Sharon D, } \\
\text { Grinberg K. } \\
\text { (2018) }\end{array}$ & odo & $\begin{array}{l}\text { Se encontró una correlación positiva entre el nivel de IE y el grado de éxito en los } \\
\text { estudios de enfermería entre los estudiantes de enfermería. Existe la necesidad } \\
\text { de aumentar la importancia de la IE en términos de la admisión de los } \\
\text { estudiantes de enfermería y los currículos básicos de enfermería }\end{array}$ \\
\hline $\begin{array}{l}\text { Oliveira } K R E \text {, } \\
\text { Trovo } M M, \\
\text { Risso } A C M \\
\text { da } C R \text {, et al. } \\
\text { (2018) }\end{array}$ & vación & $\begin{array}{l}\text { El desarrollo de las habilidades de comunicación está influenciado por factores } \\
\text { como la experiencia de las actividades prácticas, las características individuales } \\
\text { de los estudiantes, el uso de metodologías activas, el acceso a los medios de } \\
\text { comunicación, la relación de proximidad entre estudiante y profesor, y el } \\
\text { conocimiento de los conceptos teóricos de comunicación y enfermería. El uso de } \\
\text { metodologías activas parece favorecer el desarrollo de las habilidades de } \\
\text { comunicación }\end{array}$ \\
\hline $\begin{array}{l}\text { Beltrán } \\
\text { Salazar OA. } \\
\text { (2015) }\end{array}$ & $\begin{array}{l}\text { udio } \\
\text { alitativo }\end{array}$ & $\begin{array}{l}\text { Las habilidades comunicativas palabras, gestos y actitudes durante la relación } \\
\text { enfermera-paciente, en la cual la presencia, lo que se hace y la forma de hacerlo } \\
\text { permiten dejar una importante huella en los pacientes y sus familiares. }\end{array}$ \\
\hline $\begin{array}{l}\text { Ruiz } R \\
\text { Caballero }\end{array}$ & Uallitativ & $\begin{array}{l}\text { Los resultados obtenidos muestran tanto en el conjunto de habilidades } \\
\text { comunicativas y de realización de una historia clínica, como en las habilidades }\end{array}$ \\
\hline
\end{tabular}




\begin{tabular}{|c|c|c|}
\hline $\begin{array}{l}\text { D, et } \\
\text { 17) }\end{array}$ & & $\begin{array}{l}\text { pecíficas para dar malas noticias o cambiar conductas, resultados positivos } \\
\text { lucativamente significativos. }\end{array}$ \\
\hline $\begin{array}{l}\text { Tejada S, } \\
\text { Ramirez EJ, } \\
\text { Díaz RJ, } \\
\text { Huyhua SC. } \\
\text { (2018) }\end{array}$ & $\begin{array}{l}\text { Estudio } \\
\text { cualitativo, } \\
\text { exploratorio, } \\
\text { descriptivo }\end{array}$ & $\begin{array}{l}\text { Se identificaron las siguientes categorías: I) Convivencia y relaciones de } \\
\text { cuidado/descuido en la formación de enfermería; II) (Des)articulación teórico- } \\
\text { práctica en la enseñanza/aprendizaje del cuidado de enfermería: hacia la } \\
\text { integración docente-asistente; III) Complementariedad docente/estudiante para } \\
\text { la construcción de conocimiento y estrategias didácticas a través del aprendizaje } \\
\text { basado en problemas, casos hipotéticos, laboratorios vivenciales y sociodramas e } \\
\text { incluir como ejes trasversales el cuidado y la investigación. }\end{array}$ \\
\hline $\begin{array}{l}\text { Santos } B M \\
\text { dos, Silva } \\
\text { RMCRA, } \\
\text { Pereira ER, et } \\
\text { al. (2018) }\end{array}$ & tiva & $\begin{array}{l}\text { conocimiento del estudiante universitario subsidiará su práctica, guiando, } \\
\text { cilitando o dificultando su práctica en su día laboral, de acuerdo a cómo se } \\
\text { orendió. La universidad, tiene la responsabilidad de intervenir con las } \\
\text { strategias e información sobre humanización y / o prácticas humanizadas en } \\
\text { ursos de pregrado. }\end{array}$ \\
\hline $\begin{array}{l}\text { Aim A, } \\
2017\end{array}$ & 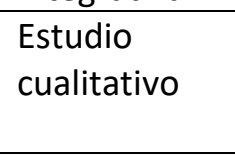 & $\begin{array}{l}\text { ra brindar atención integral al paciente y las familias, se deben adquirir las } \\
\text { mpetencias necesarias a través de la educación continua y la reflexión } \\
\text { dividual sobre la práctica personal. }\end{array}$ \\
\hline $\begin{array}{l}\text { C, } \\
\text { hani } \\
\text { hl L, } \\
\text { ez B. }\end{array}$ & & $\begin{array}{l}\text { ases teóricas, el tutorial, el seminario, la evaluación e } \\
\text { ácticas interdisciplinarias y multiprofesionales, prácti } \\
\text { rrículo en estudio favoreció el desarrollo de las habilidade }\end{array}$ \\
\hline a. & $\begin{array}{l}\text { vo, } \\
\text { nte, }\end{array}$ & $\begin{array}{l}\text { Los resultados señalan la relevancia de estudiar el proceso de la comunicación, } \\
\text { pues, practicándola, hay una mejora en la forma en que el enfermero se } \\
\text { comunica, evitando interferencias que puedan poner en peligro las } \\
\text { informaciones ofrecidas. Se destaca la importancia de la relación } \\
\text { enfermero/paciente, que permite la comprensión de las informaciones } \\
\text { educativas y la forma en que se prestan, constituyendo poderosa herramienta }\end{array}$ \\
\hline $\begin{array}{l}\text { Siles- } \\
\text { González, J., } \\
\text { Noreña- } \\
\text { Peña, A.L. y } \\
\text { Solano-Ruiz, } \\
\text { C. (2017) }\end{array}$ & 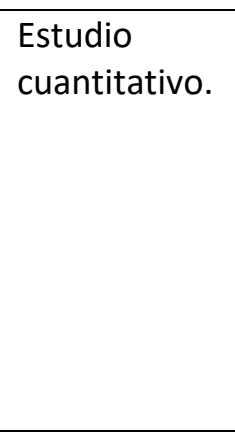 & $\begin{array}{l}\text { Se deben desarrollar estrategias innovadoras para la toma de conciencia, } \\
\text { reflexión, valoración y análisis de las emociones y sentimientos derivados de } \\
\text { actividades desarrolladas por alumnos del grado de enfermería durante sus } \\
\text { prácticas clínicas. El abordamiento exclusivamente psicométrico de la inteligencia } \\
\text { emocional no parece la solución más pertinente y solvente. Para que el } \\
\text { aprendizaje de las emociones y sentimientos resulte significativo para el } \\
\text { educando, es precisa la implicación del mismo mediante procesos de toma de } \\
\text { conciencia en los que se clarifique la importancia de dimensiones como: la } \\
\text { metacognición, la reflexión en la acción, el pensamiento crítico y la estética. }\end{array}$ \\
\hline A, & & $\begin{array}{l}\text { con los } \\
\text { ue más } \\
\text { tipo de }\end{array}$ \\
\hline $\begin{array}{l}\text { Guerrero R, } \\
\text { Meneses } M \text {, } \\
\text { De La Cruz } \\
\text { M. (2016) }\end{array}$ & y & $\begin{array}{l}\text { intar } \\
\text { erar } \\
\text { ente }\end{array}$ \\
\hline $\begin{array}{l}\text { Rojas MM, } \\
\text { González ME } \\
\text { (2018) }\end{array}$ & $\begin{array}{l}\text { Estudio } \\
\text { histórico- }\end{array}$ & $\begin{array}{l}\text { El desarrollo de habilidades comunicativas constituye un requerimiento esencial } \\
\text { para garantizar eficiencia en los servicios de salud. Las dificultades de desarrollo } \\
\text { apuntan a la necesidad de que se tome en cuenta la concepción desarrolladora } \\
\text { de la educación que se promueve actualmente al abordarse los procesos } \\
\text { formativos universitarios de las ciencias médicas. }\end{array}$ \\
\hline & & $\begin{array}{l}\text { La intervención educativa fue efectiva para aumentar el conoc } \\
\text { recomienda que el personal de enfermería continúe capacitado en }\end{array}$ \\
\hline
\end{tabular}




\begin{tabular}{|c|c|c|}
\hline et al. (2018) & & los servicios hospitalarios. \\
\hline $\begin{array}{l}\text { Hobson WL, } \\
\text { Hoffmann K, } \\
\text { Loue S, et al. } \\
\text { (2018) }\end{array}$ & $\begin{array}{l}\text { Estudio } \\
\text { cuantitativo }\end{array}$ & $\begin{array}{l}\text { Los participantes informaron de que su nivel de confianza en el uso de técnicas } \\
\text { teatrales como una herramienta para la educación médica aumentó de la } \\
\text { pretesión de confianza baja a media a la postesión de alta confianza. Todos los } \\
\text { encuestados que estaban enseñando activamente dijeron que habían hecho } \\
\text { cambios en su enseñanza y que apreciaron el aprendizaje activo en la sesión. }\end{array}$ \\
\hline $\begin{array}{l}\text { Reuter } C, \\
\text { Santos V, } \\
\text { Ramos A, et } \\
\text { al. (2018) }\end{array}$ & $\begin{array}{l}\text { Estudio } \\
\text { exploratorio, } \\
\text { cualitativo }\end{array}$ & $\begin{array}{l}\text { Se destaca la efectividad de acciones interprofesionales e intersectoriales. } \\
\text { Posibilitan la realización de acciones en salud de forma integral y holística, } \\
\text { optimizando las prácticas de cuidados en Enfermería. }\end{array}$ \\
\hline $\begin{array}{l}\text { Moraes } M \text {, } \\
\text { Tarcila P, } \\
\text { Rêgo Lopes } \\
\text { TM (2019) }\end{array}$ & $\begin{array}{l}\text { Estudio } \\
\text { cualitativo }\end{array}$ & $\begin{array}{l}\text { Cuando hay una efectiva comunicación y una buena relación interpersonal, la } \\
\text { familia se siente más segura y más cómoda. Se observó que la comunicación de } \\
\text { salud, es una excelente herramienta de trabajo en el cuidado de la salud porque } \\
\text { promueve mayor interacción, facilitando la creación de enlaces confianza y } \\
\text { obtener un mejor grado de satisfacción en los servicios ofrecidos tanto por el } \\
\text { cliente como por el Servicio de personal. Además, los miembros de la familia se } \\
\text { sienten arropados cuando los profesionales muestran sentimientos de respeto, } \\
\text { afecto, comprensión y atención, y ese cuidado trasciende la realización de } \\
\text { procedimientos. Sensibilizar a los profesionales sobre importancia de la presencia } \\
\text { familiar es el primer paso hacia el cambio. Conocer la percepción de la familia } \\
\text { sobre el cuidado de enfermería es esencial para que se realicen acciones más } \\
\text { acogedoras que ayudan a la familia a sobrellevar la hospitalización de un familiar. }\end{array}$ \\
\hline $\begin{array}{l}\text { Villar } F \\
\text { Serrat } R \text {. } \\
\text { (2017) }\end{array}$ & $\begin{array}{l}\text { ón } \\
\text { ria }\end{array}$ & $\begin{array}{l}\text { Describe la relevancia del enfoque narrativo para la comprensión de la } \\
\text { experiencia de la persona mayor que es cuidada en instituciones, tanto en sus } \\
\text { aspectos individuales como en las relaciones que establece con los profesionales, } \\
\text { y en los discursos institucionales que contextualizan esas relaciones. En segundo } \\
\text { lugar, especifica diversas formas en las que el uso de narrativas podría tener un } \\
\text { impacto en la mejora de la calidad de la atención y el bienestar de las personas } \\
\text { mayores que reciben cuidados en instituciones. }\end{array}$ \\
\hline $\begin{array}{l}\text { Siles } \\
\text { González J } \\
\text { (2018) }\end{array}$ & $\begin{array}{l}\text { tal } \\
0 \\
0\end{array}$ & $\begin{array}{l}\text { La más importante aportación de la poesía a la enfermería radica en la } \\
\text { clarificación de su naturaleza transcendente mediante la revelación de su objeto } \\
\text { sujeto: el ser humano. La persona como núcleo, como materia prima con la que } \\
\text { trabaja la enfermera para "producir su obra" }\end{array}$ \\
\hline $\begin{array}{l}\text { Guillaumet } \\
\text { M, Amorós } \\
\text { G, Ramos A, } \\
\text { et al. (2017) }\end{array}$ & $\begin{array}{l}\text { io } \\
\text { ativo }\end{array}$ & $\begin{array}{l}\text { Tomar consciencia de la propia vivencia en torno a la muerte permite precisar } \\
\text { aspectos significativos que pueden enriquecer los cuidados profesionales. El } \\
\text { relato pedagógico, como estrategia didáctica, es útil en la formación enfermera } \\
\text { ya que posibilita la comprensión de significados y del impacto ante situaciones } \\
\text { complejas. La reflexión sobre las experiencias vividas y el contraste con la } \\
\text { literatura permite guiar el proceso del cuidado comprensivo. }\end{array}$ \\
\hline $\begin{array}{l}\text { Ceballos } L M \text {, } \\
\text { Rodríguez } \\
\text { LA, } \\
\text { González SC } \\
\text { (2018) }\end{array}$ & bibliográfica & $\begin{array}{l}\text { Se concluyó que las últimas tendencias de la educación superior demandan un } \\
\text { cambio, desde un paradigma instructivo a uno cognitivo, desarrollado por el } \\
\text { propio estudiante, en el que la investigación cualitativa, junto a la cuantitativa } \\
\text { constituyan el eje central en la construcción del conocimiento científico; la } \\
\text { puesta en práctica de habilidades investigativas debe incluir investigación } \\
\text { cualitativa, para lograr práctica profesional superior a la altura de los } \\
\text { requerimientos sociales actuales }\end{array}$ \\
\hline $\begin{array}{l}\text { Gomes G, } \\
\text { Xavier D, } \\
\text { Pintanel A, et } \\
\text { al. (2015) }\end{array}$ & $\begin{array}{l}\text { tudio } \\
\text { alitativo. }\end{array}$ & $\begin{array}{l}\text { Las interacciones entre los miembros de la familia y el equipo de enfermería } \\
\text { contribuyen a la importancia atribuida por la familia al cuidado de enfermería } \\
\text { recibido por el niño. Las enfermeras deben ser conscientes de las actitudes del } \\
\text { equipo de enfermería con respecto al niño y su familia, priorizando la atención } \\
\text { humanizada. }\end{array}$ \\
\hline $\begin{array}{l}\text { Azevêdo AV, } \\
\text { Launchoni }\end{array}$ & $\begin{array}{l}\text { evisión } \\
\text { bliográfica }\end{array}$ & $\begin{array}{l}\text { En el establecimiento de relaciones interpersonales, tanto técnica como } \\
\text { formalmente, existen dificultades en las acciones de comunicación y atención. La }\end{array}$ \\
\hline
\end{tabular}


De los estudios seleccionados se han extraído cuatro categorías de análisis: 1) las habilidades comunicativas y emocionales como elementos esenciales del cuidado humanizado, 2) la necesaria capacitación emocional y comunicativa en la formación de enfermería, 3) otros factores que contribuyen a un cuidado humanizado y 4) cambios metodológicos y recursos pedagógicos para la capacitación comunicativa y emocional de docentes, alumnado y profesionales de enfermería.

\section{Las habilidades comunicativas y emocionales: elementos esenciales del Cuidado Humanizado.}

Son numerosos los estudios que corroboran la importancia de mejorar la comunicación en los procesos de cuidado para el establecimiento de una relación interpersonal enfermero-paciente ${ }^{(5)}$. El tipo de relación y el tipo de cuidados que se establezca entre el profesional de la enfermería y el usuario pueden tener efectos terapéuticos, aumentado el grado de aceptación y tolerancia del tratamiento médico y los procedimientos de enfermería ${ }^{(3)}$.

Esto lo podemos comprobar en los resultados del reciente estudio cualitativo desarrollado por Michelan y Spiri(6). Entre las conclusiones, se destaca la necesidad de la asistencia holística, lo cual repercute de manera positiva en la recuperación del paciente, pues refieren que el diálogo y la escucha son comparables con un tratamiento medicamentoso.

Cuando las personas se sienten bien atendidas, escuchadas y respetadas y mantienen un vínculo de confianza tienden a cumplir más el tratamiento. En esta línea, se encuentran estudios que relacionan el entrar en la esfera emocional del paciente y favorecer la expresión de experiencias emocionales, con una mejor satisfacción, recuperación, e incluso, ser determinantes en el proceso de sanación ${ }^{(6,7)}$. Por el contrario, encontramos que una comunicación ineficaz por parte del profesional sanitario puede causar depresión, aumentar la ansiedad, indefensión, desesperanza y empeorar la calidad de vida de los pacientes.

En este sentido, Pott et al. ${ }^{(3)}$, señalan como factores que empobrecen la calidad del cuidado, por un lado, la ineficacia y/o la ausencia de comunicación, en sus distintas formas durante las acciones de cuidado, descuidando la dimensión emocional y social de la persona necesitada de cuidados. Y, por otro lado, la mecanización y rutinización de los cuidados diarios, lo que evidencia que muchos profesionales se centran en el tratamiento y cura del cuerpo enfermo, sin considerar las demás dimensiones humanísticas que conforman a cada ser. Sin embargo, en la bibliografía al respecto, se desvela que entre los usuarios crece la demanda de un trato más humano y no sólo la solicitud de un tratamiento. Los usuarios perciben que la relación interpersonal y el tipo de trato que se establece entre el enfermero/a-paciente son las características más valoradas, incluso por encima de la eficacia. La inteligencia emocional debe ser uno de los objetivos de la formación y práctica sanitaria, y que debe ser reconocida entre los indicadores para mejorar la calidad de los servicios de salud, en tanto que las personas que poseen altos índices de inteligencia emocional son más capaces de empatizar con los demás, son más propensos a prestar ayuda, a 
cooperar y a establecer relaciones interpersonales más satisfactorias. Asimismo, es preciso destacar la actual investigación realizada por Sharon y Griberg ${ }^{(8)}$, centrada en revisar la relación entre el nivel de inteligencia emocional y el grado de éxito de los estudiantes en enfermería, cuyos resultados señalan una correlación positiva entre estas variables, sosteniendo que la inteligencia emocional debe considerarse como criterio para la admisión de estudiantes a los programas de estudios de pregrado en enfermería.

Lo hasta aquí presentado pone de relieve la importancia de los cuidados emocionales y buen trato que, a través del establecimiento de relaciones interpersonales de confianza, con especial atención al tipo de comunicación, pueden convertirse en un elemento terapéutico que influye en el bienestar, la salud y/o proceso de recuperación de las personas necesitadas de cuidados.

\section{Otros factores que contribuyen a un cuidado humanizado: atención, reflexión y autoconocimiento.}

Existen otros factores que contribuyen a desarrollar un cuidado humanizado y que dependen directamente del profesional. La forma de ser de la persona y su carácter influye directamente en el trato al paciente, tanto en la forma de dirigirse al paciente y su familia como en su comportamiento, alentando o descuidando una práctica más o menos humanizada ${ }^{(9)}$.

De ahí que la reflexión de la práctica enfermera en el cuidado diario se haga imprescindible. Según estos autores para alcanzar la humanización del cuidado es necesario un proceso reflexivo permanente que permita tener presente los valores y principios que rigen la práctica profesional, ya que la enfermería, por sí misma, es una práctica humanizada.

Situamos aquí la teoría del Cuidado Humano de Jean Watson, en tanto que desde esta teoría se le otorga una especial importancia a la reflexión sobre el propio yo, a la introspección personal y/o a la autoconciencia como factor del proceso de cuidado. Watson (10) afirma que una persona que no reconoce sus propios sentimientos, difícilmente logrará entender los de otras personas. Al mismo tiempo, la aceptación de la expresión de los sentimientos propios y ajenos (tanto positivos como negativos), permite una empatía que transciende el propio yo y que favorece el proceso de cuidado humano(11). De ahí que el autoconocimiento, la práctica reflexiva sobre el cuidado que se realiza y la capacidad de motivación, sean elementos necesarios que incorporar en la profesionalización y formación del personal de enfermería(12).

Otra de las cuestiones que se presentan como esenciales para un cuidado humanizado es "el detalle en la atención". Beltrán-Salazar ${ }^{(13)}$, en su estudio cuyo propósito era comprender el sentido del cuidado humanizado en la experiencia de personas hospitalizadas, familiares de pacientes y enfermeras, señala la importancia del detalle y cómo éstos pueden ser percibidos por las personas necesitadas de cuidados. Este autor concluye que, si bien para las enfermeras puede no revestir importancia realizar ciertas acciones o tener en cuenta determinados detalles, para las personas necesitadas de cuidados pueden ser esenciales detalles como el saludo, dirigirse a la persona por su nombre, las miradas de complicidad, conocer sus gustos, contacto corporal con caricias, en tanto que son elementos importantes en la forma de la interacción y en el establecimiento de una relación interpersonal basada en la confianza desde el cuidado humanizado. 
Se comprueba, por tanto, que la atención al detalle, la práctica reflexiva y el autoconocimiento, se presentan como factores que contribuyen a un cuidado humanizado.

\section{La capacitación emocional y comunicacional en la formación de la enfermería para un cuidado humanizado}

Actualmente, los planes formativos en el contexto español contemplan las competencias emocionales y de comunicación dentro del conjunto de competencias que el estudiante de enfermería debe alcanzar para obtener el título de Graduado en Enfermería ${ }^{(4)}$. La comunicación clínica ha sido aceptada como un contenido importante de los planes de estudio de enfermería y reconocida como una competencia esencial por los organismos de acreditación y las organizaciones internacionales ${ }^{(14)}$.

Pese a ello, se señala el estancamiento que ha experimentado la formación de habilidades comunicativas y emocionales en la enfermería Son, incluso, los propios profesionales de la enfermería quienes destacan que aprender a escuchar, gestionar y comunicar las emociones tendría que ser una habilidad que se debería enseñar durante la formación básica de manera transversal. Al respecto, se señala que esta formación debe desarrollarse desde nuevos enfoques y metodologías más participativas y experienciales que aseguren un aprendizaje significativo ${ }^{(15)}$.

De acuerdo con Santos et al. ${ }^{(16)}$ la deshumanización comienza en la formación de la enfermería, donde se mantiene el énfasis en el dominio de conocimientos, evidencias científicas y el desarrollo de habilidades técnicas, en detrimento de habilidades y competencias centradas en el paciente desde una mirada integral, ignorado el enfoque crítico, creativo y sensible-afectivo inherente al cuidado. A similares conclusiones llegan Lee, et al.(11), quienes evidencian la necesidad de incorporar formación específica en aspectos emocionales con metodologías que potencien y promuevan más el aprendizaje reflexivo, para alcanzar las competencias necesarias para una atención integral. En consecuencia, estos autores manifiestan que "la formación de los estudiantes debe privilegiar la comunicación con la persona antes que el desempeño del rol técnico, en la medida que sean capaces de clarificar las necesidades y sentimientos de cada persona sujeto de cuidado". (p.40).

Cabe destacar, también, el actual interés formativo relativo al estudio de la presencia y atención de los familiares, como un aspecto de influencia directa en la recuperación y mejora de las personas necesitada de cuidados, siendo un aspecto esencial en el cuidado humanizado (17). En esta línea se sitúan los resultados de Gomes, at al. (18), desde los que se desprende que la presencia de los familiares es un elemento que potencia la recuperación, tanto física como emocional, de la persona cuidada, sobre todo cuando se trata del cuidado infantil. Se pone de manifiesto que, entre los significados de humanización, la incorporación de la familia dentro del cuidado al paciente debe ser reconocida como práctica inherente al profesional de enfermería para proporcionar un cuidado más completo, holístico y humano ${ }^{(19)}$. En consecuencia, se sugiere como necesario que el cuidado familiar sea incluido en los programas formativos de enfermería, contribuyendo a que el personal en formación adquiera habilidades y competencias para trabajar con los familiares; y sobre todo, que puedan comprender la importancia de incluir a la familia en el plan de atención ${ }^{(20)}$. 
No obstante, no es suficiente con incorporar la formación en los grados académicos, sino que debiera ser una formación continua, que acompañe al profesional de enfermería durante toda su trayectoria profesional. Al respecto, Tejada, et al. ${ }^{(15)}$ insisten en la necesidad de una actualización pedagógica y profesional permanente que contribuya a la integración de estrategias innovadoras para un aprendizaje significativo. A similares conclusiones llega el estudio de Guerrero-Ramírez, MenesesLa Riva y De la Cruz-Ruiz (21), en las que reclaman la necesidad de implementar estrategias, planes de mejora y capacitación continua con la finalidad de generar sensibilización del personal de enfermería para aplicar un cuidado humanizado en la atención al paciente, otorgándole a la comunicación, al acompañamiento, a la relación con el paciente-familia, y a la empatía, la máxima importancia.

La Universidad debe ser tan científica como sensible(22), y debe generar los cambios necesarios que permitan asegurar que los egresados de nuestras facultades, adquieren las competencias y habilidades necesarias para saber brindar un cuidado humanizado. En este sentido, siguiendo a Ruiz-Moral, et al.(14), cabe destacar las modificaciones curriculares que se llevaron a cabo en la Facultad de Medicina de Francisco de Vitoria (España), en la que se implementaron ciertas particularidades de tipo organizativo que han facilitado la integración de habilidades de comunicación y cuidado de manera transversal.

Por su parte, destacamos aquí los resultados del estudio de Hernández et al. (23), desde el que se implementó una intervención educativa con el objetivo de mejorar el uso del indicador trato digno en el personal de enfermería que participa en el cuidado hospitalario del paciente. El propósito del estudio fue analizar si la formación se veía reflejada en el cuidado y la relación con el paciente. Sobre los resultados de las evaluaciones antes y después, las medias obtenidas aumentaron significativamente. Se concluye que la intervención educativa tuvo un impacto importante en la obtención de conocimientos del personal de enfermería, así como en la mejora de la implementación del indicador.

Lo escrito hasta aquí, pone de manifiesto que realizar cambios y mejoras en el tratamiento y formación de las habilidades de comunicación y emocionales, constituye una necesidad que debe ser priorizada, tanto en el ámbito académico e investigador como en el profesional, al ser elementos vehiculares para alcanzar la calidad de la atención.

\section{Estrategias y recursos educativos para la capacitación comunicativa de docentes, estudiantes y profesionales sanitarios.}

El alumnado manifiesta sentirse poco o nada preparado para afrontar las exigencias comunicativas y emocionales. Asimismo, las evidencias aportadas por las investigaciones avalan las ideas que venimos defendiendo. Los resultados científicos reivindican la necesidad de mejorar la capacitación de los futuros profesionales de la enfermería con respecto a las competencias comunicativas y emocionales. Sin embargo, se señala que la adquisición y desarrollo de estas competencias requieren de un trabajo especial. El simple hecho de proporcionar conocimientos de forma expositiva, siguiendo el modelo de clases magistrales y ofreciendo lecciones o seminarios de forma puntual sobre estas competencias, puede no ser suficiente para ayudar al estudiantado a desarrollar la empatía y las habilidades de comunicación necesarias para brindar una educación y cuidado eficiente y afectivo(14). En la bibliografía se reclama la incorporación de nuevos enfoques metodológicos que 
reemplacen la insuficiencia de las clases expositivas y contribuyan a generar procesos de aprendizaje significativos(24). Al respecto, Tejada, et al. ${ }^{(15)}$ señalan la necesidad de integrar el aprendizaje a partir de metodologías participativas, reflexivas y dialógicas que respeten la autonomía de los estudiantes y el trabajo en equipo para construir, reconstruir e intercambiar saberes. Los resultados de su estudio inciden en que las prácticas pedagógicas juegan un rol esencial en los procesos de enseñanzaaprendizaje sobre el cuidado en los futuros profesionales de enfermería, al contribuir en el desarrollo de una sensibilidad humana especial, al despertar el enfoque afectivo, creativo y crítico. A similares resultados llega de Oliveira, et al. ${ }^{(9)}$ en su estudio, cuyo objetivo fue comprender, desde la perspectiva de los docentes, cuáles son las facilidades y dificultades en el desarrollo de habilidades comunicativas en estudiantes de enfermería. Se destacan como factores que contribuyen al aprendizaje: uso de actividades prácticas, características individuales de los estudiantes, uso de metodologías activas ${ }^{(25)}$, relación de proximidad de profesores con alumnos y organización en pequeños grupos .

En consonancia con lo anterior, destacamos las recientes aportaciones de Rojas y Gonzáles $^{(22)}$, quienes señalan que el proceso de enseñanza-aprendizaje participativo, adquiere un valor añadido cuando su finalidad es el desarrollo de habilidades comunicativas, en tanto que su adquisición requiere de escenarios formativos en los que el intercambio, la reflexión y la influencia mutua sean el eje vehicular dinámico de la práctica educativa. Como insisten estos autores, se deben estimular espacios y entornos pedagógicos donde predomine la reflexión personal, el intercambio de opiniones y la discusión abierta y respetuosa, favorecedora de aprendizajes significativos y de desarrollo personal y colectivo vinculados al proceso de comunicación.

Otro de los elementos a integrar en los procesos de aprendizaje que señala la bibliografía para mejorar las habilidades y competencias emocionales y de comunicación, es el trabajo interdisciplinario. Los resultados de un reciente estudio, indican que el $90 \%$ de los estudios evidencian que la formación interprofesional es indispensable en estudiantes de salud(22). Tanto es así que, Naciones Unidas en colaboración con la Organización Mundial de la Salud y otros organismos, propusieron en 2016 la educación interprofesional e interdisciplinaria en la formación inicial, enfocándose en potenciar una educación transformadora con calidad y competencias integradas de todos los campos.

Estudios recientes, apuntan que el trabajo interdisciplinario puede favorecer una multiplicidad de enfoques y alternativas para comprender los aspectos que están involucrados en el cuidado del paciente, contribuyendo al establecimiento de una nueva cultura de la atención enfermera, lo cual debe ser trasladado al ámbito formativo(26).

Otro de los recursos que se plantean para contribuir a un cambio en las prácticas enfermeras hacia un cuidado humanizado, que mejoren la formación en competencias comunicativas y emocionales centrado en la persona y su familia, es la narración, siendo especialmente relevantes las narrativas autobiográficas ${ }^{(27)}$.

Se sitúa aquí la autoetnografía. El uso de la escritura de la propia historia como una forma de entender los procesos vitales propios y de nuestros pacientes, permite reflexionar, crear y aprender como personas y como profesionales de la enfermería. Un proceso autoetnográfico, requiere ejercitar la observación de forma consciente, 
manteniendo la atención en la conexión con el paciente, en cómo nos reflejamos en ellos, de qué manera interactuamos y nos vinculamos en las relaciones interpersonales y si ponemos en práctica experiencias, habilidades y aprendizajes en los que la comunicación efectiva y afectiva sea el eje vehicular de la interacción con el otro. De este modo, se presenta como un recurso para trabajar nuestros sentimientos, nuestra formas de comunicarnos y de relacionarnos, a partir de nuestra propia historia y de la de los otros, para así comprenderla y acercarnos a comprender a los otros ${ }^{(28)}$.

Estas contribuciones en el profesional de enfermería también ocurren cuando se utiliza este método en pacientes y sus propias vivencias de enfermedad, lo que permite expresar sus sentimientos y emociones, y compartir con otros su experiencia, contribuyendo de manera directa a mejorar el cuidado personal de pacientes, así como también a que las enfermeras comprendan la posición de la persona necesitada de cuidados.

Considerando lo mencionado, es posible afirmar que las metodologías cualitativas, narrativas e interpretativas nos obligan a (re)cuestionar constantemente nuestras concepciones sobre los seres humanos, nuestros problemas personales, sociales, físicos, mentales, y espirituales; lo que influye de manera directa en nuestra posición en relación con el otro ${ }^{(29)}$.

Por último, es preciso destacar que, a pesar del desarrollo positivo de estas metodologías en la investigación en salud, también se señala como un obstáculo el carácter subjetivo y de expresión de sentimientos de esta metodología en el encuentro con la ciencia más cercana a la objetividad. Sin embargo, como sostiene Siles(28), para tratar de dar respuesta a esta barrera, ha surgido la sociopoética que permite el estudio de la expresión de los sentimientos de forma intersubjetiva alcanzando de esa forma cierta objetividad. Diversos autores han descrito la utilidad de la poesía en enfermería.

Asimismo, también se hace mención a la posibilidad de auto-narrativas poco profundas en el plano interpretativo, lo cual puede resultar contraproducente al descuidar la misión principal de conectar lo personal con lo social de la autoetnografía. De ahí, que algunos autores hayan puesto sus esfuerzos en intentar proporcionar pautas para ubicar este método de investigación, dentro del marco de una buena investigación social, señalando la construcción de un modelo Educativo Basado en la Narración (EBN) ${ }^{(28,30)}$, desde el que se sugiere que pueda servir como puente entre la subjetividad cualitativa de este modelo y la objetividad científica de la EBE (Educación Basada en la Evidencia).

\section{CONCLUSIONES}

Desde la década de los 80 , en la que el paradigma de la transformación comienza a desarrollarse, son muchos los reclamos que centran su atención en recuperar el enfoque humano en las instituciones de salud. Sin embargo, tras la revisión bibliográfica realizada, se evidencia que, en la actualidad, continuamos investigando y reclamando tal enfoque para un cuidado humanizado, ya que, tanto en la práctica profesional de la enfermería como en la formación, se mantiene el paradigma neopositivista, provocando el limitado enfoque de la enfermería al ámbito del método experimental, cuantitativo y objetivo, alejándola de su auténtico epicentro 
epistemológico: el cuidado humanizado. Al respecto, se señala la importancia y necesidad de clarificar y entender las características que contempla el cambio de paradigma que venimos sosteniendo, desde el que se reclama la adopción de teorías y metodologías participativas y cualitativas, donde la reflexión, la profundidad y la subjetividad adquieren valor con la intención de mejorar la capacitación en competencias comunicativas y emocionales, siendo elementos esenciales para el cuidado humanizado. Asimismo, se insiste en la importancia de otros factores como son la atención al detalle, la reflexión y el autoconocimiento, como elementos que contribuyen a la humanización de los cuidados.

Asumir y llevar a la práctica lo escrito hasta aquí, en las circunstancias y realidades actuales, implica la adopción de un nuevo enfoque formativo y práctico. En la literatura analizada, se reclama la necesidad de incorporar, de manera más pedagógica y profunda, programas formativos en competencias emocionales y de comunicación dentro de la formación, tanto del grado de enfermería como a lo largo de su trayectoria profesional.

Al respecto, se plantea la formación interdisciplinar y narración como metodología investigativa y pedagógica que puede contribuir a la adquisición y desarrollo de tales competencias. Se propone el uso de la escritura de la propia historia, como una forma de entender los procesos vitales propios y de nuestros pacientes, lo que permite reflexionar y aprender en y desde nuestros procesos vitales y experienciales como personas y como profesionales de la enfermería. Es a través del autoconocimiento desde donde se pone en marcha y se posibilita la revisión, reflexión y transformación de las propias creencias, pensamientos y formas de actuar, enfocándose hacia una atención más integral.

\section{REFERENCIAS}

1. Mastrapa YE, Lamadrid M del PG. Relación enfermera-paciente: una perspectiva desde las teorías de las relaciones interpersonales. Rev Cubana Enferm [Internet]. 2016 Dec 29 [cited 2019 Jul 19];32(4). Available from: http://www.revenfermeria.sld.cu/index.php/enf/article/view/976/215

2. Cestari VRF, Moreira TMM, Pessoa VLM de P, Florêncio RS, Silva MRF da, Torres RAM, et al. The essence of care in health vulnerability: a Heideggerian construction. Rev Bras Enferm [Internet]. 2017 Oct [cited 2019 Jul 20];70(5):1112-6. Available from:http://www.scielo.br/scielo.php?script=sci_arttext\&pid=S0034-

71672017000501112\&lng=en\&tIng=en

3. Pott FS, Stahlhoefer T, Felix JVC, Meier MJ. Medidas de conforto e comunicação nas ações de cuidado de enfermagem ao paciente crítico. Rev Bras Enferm [Internet]. 2013 Apr;66(2):174-9. Available from: http://www.scielo.br/scielo.php?script=sci_arttext\&pid=S0034$71672013000200004 \&$ Ing=pt\&tIng=pt

4. Minguez-Moreno, I., Siles J. Pensamiento crítico en enfermería: de la racionalidad técnica a la práctica reflexiva. Aquichan [Internet]. 2014;14(4):594-604. Available from: http://www.scielo.org.co/pdf/aqui/v14n4/v14n4a13.pdf

5. Oliveira AM de, Soares E. A Comunicação como Ferramenta Educativa no PréOperatório Mediato de Transplante Renal pesquisa, cuidado é fundamental online. Rev Pesqui Cuid é Fundam [Internet]. 2009 Sep 26 [cited 2019 Jul 29];753-7. Available from: http://ciberindex.com/index.php/ps/article/view/P103753

6. Michelan VC de A, Spiri WC. Perception of nursing workers humanization under 
intensive therapy. Rev Bras Enferm [Internet]. 2018 Apr;71(2):372-8. Available from: http://www.scielo.br/scielo.php?script=sci_arttext\&pid=S0034-

$71672018000200372 \&$ Ing=en\&tIng=en

7. Uno M, Tsujimoto T, Inoue T. Perceptions of nurses in Japan toward their patients' expectations of care: A qualitative study. Int J Nurs Sci [Internet]. 2017 Jan 10 [cited 2019 Jul 18];4(1):58-62. Available from: https://www.sciencedirect.com/science/article/pii/S235201321630093X

8. Sharon D, Grinberg K. Does the level of emotional intelligence affect the degree of success in nursing studies? Nurse Educ Today [Internet]. 2018 May;64:21-6. Available

from:https://www.sciencedirect.com/science/article/pii/S0260691718300583?via\%3Dih $\mathrm{ub}$

9. Oliveira KRE de, Trovo MM, Risso ACM da CR, Braga EM, Oliveira KRE de, Trovo $\mathrm{MM}$, et al. The teaching approach on communicative skills in different teaching methodologies. Rev Bras Enferm [Internet]. 2018 Oct [cited 2019 Jul 29];71(5):244753. Available from: http://www.scielo.br/scielo.php?script=sci_arttext\&pid=S003471672018000502447\&lng=en\&tIng=en

10. Watson J. Watson's theory of human caring and subjective living experiences: carative factors/caritas processes as a disciplinary guide to the professional nursing practice. Texto Context - Enferm [Internet]. 2007 Mar [cited 2019 Jul 18];16(1):129-35. Available from:http://www.scielo.br/scielo.php?script=sci_arttext\&pid=S010407072007000100016\&lng=en\&tIng=en

11. Lee $H$, Kim A, Meong A, Seo M. Pediatric nurse practitioners' clinical competencies and knowing patterns in nursing: Focus group interviews. Contemp Nurse [Internet]. $2017 \quad$ Sep;53(5):515-23. Available from: https://www.tandfonline.com/doi/full/10.1080/10376178.2017.1315827

12. Siles-González J, Noreña-Peña AL, Solano-Ruiz C. Emotional intelligence during clinical practices of nursing students: an approach from educational anthropology applied to clinical practices. Ene [Internet]. 2017 [cited 2019 Jul 30];11(2):0-0. Available

from:http://scielo.isciii.es/scielo.php?script=sci_arttext\&pid=S1988348X201700020000 8

13. Beltrán Salazar OA. Humanized care: A relationship of familiarity and affectivity. Investig y Educ en Enfermería [Internet]. 2015 Feb;33(1):17-27. Available from: https://aprendeenlinea.udea.edu.co/revistas/index.php/iee/article/view/21813/17979

14. Ruiz Moral R, Caballero Martínez F, García de Leonardo C, Monge D, Cañas F, Castaño P. Enseñar y aprender habilidades de comunicación clínica en la Facultad de Medicina. La experiencia de la Francisco de Vitoria (Madrid) [Internet]. Vol. 18, Educacion Medica. Elsevier; 2017 [cited 2019 Jul 18]. p. 289-97. Available from: https://www.sciencedirect.com/science/article/pii/S1575181317300736?via\%3Dihub 15. Tejada S, Ramirez EJ, Díaz RJ, Huyhua SC. Práctica pedagógica en la formación para el cuidado de enfermería. Enfermería Univ [Internet]. 2019 Jan 25 [cited 2019 Jul 18];16(1). Available from: http://www.revistaenfermeria.unam.mx:80/ojs/index.php/enfermeriauniversitaria/article/view/577

16. Santos BM dos, Silva RMCRA, Pereira ER, Joaquim FL, Goés TRP, Santos BM dos, et al. Nursing students' perception about humanized care: an integrative review. Rev Bras Enferm [Internet]. 2018 [cited 2019 Jul 18];71(suppl 6):2800-7. Available from:

http://www.scielo.br/scielo.php?script=sci_arttext\&pid=S003471672018001202800\&lng=en\&tIng=en

17. Azevêdo AV dos S, Lançoni Júnior AC, Crepaldi MA. Interação equipe de enfermagem, família, e criança hospitalizada: revisão integrativa. Cien Saude Colet [Internet]. 2017 Nov [cited 2019 Jul 30];22(11):3653-66. Available from: 
http://www.scielo.br/scielo.php?script=sci_arttext\&pid=S1413-

81232017021103653\&lng=pt\&tlng=pt

18. Gomes GC, Xavier DM, Pintanel AC, Farias DHR, Lunardi VL, Aquino DR. Meanings attributed by family members in pediatrics regarding their interactions with nursing professionals. Rev da Esc Enferm [Internet]. 2015 Dec [cited 2019 Jul 30];49(6):951-7.

Available

from:

http://www.scielo.br/scielo.php?script=sci_arttext\&pid=S008062342015000600951\&ln $\mathrm{g}=\mathrm{en} \&$ tIng=en

19. Tavares MM e M, Coelho PTG, lopes thalyta mariany rêgo. Perception of the family on nursing assistance in an adult intensive therapy unit. Rev Enferm da UFPI [Internet]. 2019 Apr 7 [cited 2019 Jul 30];8(1):17-22. Available from: https://ojs.ufpi.br/index.php/reufpi/article/view/7445/pdf

20. Murillo Pérez MA, López López C, Torrente Vela S, Morales Sánchez C, Orejana Martín M, García Iglesias $\mathrm{M}$, et al. Percepción de las enfermeras sobre la comunicación con la familia de pacientes ingresados en un servicio de medicina intensiva. Enfermería Intensiva [Internet]. 2014 Oct 1 [cited 2019 Jul 20];25(4):137-45. Available from: https://linkinghub.elsevier.com/retrieve/pii/S1130239914000728

21. Guerrero R, Meneses M, De La Cruz M. Cuidado humanizado de enfermería según la teoría de Jean Watson, servicio de medicina del Hospital Daniel Alcides Carrión. LimaCallao, 2015. Rev Enferm Hered [Internet]. 2016 Feb 20 [cited 2019 Jul 18];9(2):133.

Available

from: http://www.upch.edu.pe/vrinve/dugic/revistas/index.php/RENH/article/view/3017/2967

22. Rojas Izquierdo,María Miladis, González Escalona ME. Las habilidades comunicativas en el proceso formativo del profesional de la salud. Educ Médica Super [Internet]. 2018 Apr 6 [cited 2019 Jul 18];32(3). Available from: http://ems.sld.cu/index.php/ems/article/view/1511/706

23. Hernández Terrazas LE, Díaz Oviedo A, Martínez Licona JF, Gaytan Hernandez D. Nursing education in humanized care. Esc Anna Nery [Internet]. 2018 Feb 5 [cited 2019 Jul 18];22(1). Available from: http://www.scielo.br/scielo.php?script=sci_arttext\&pid=S1414-

81452018000100219\&lng=en\&tIng=en

24. Hobson WL, Hoffmann-Longtin K, Loue S, Love LM, Liu HY, Power CM, et al. Active Learning on Center Stage: Theater as a Tool for Medical Education. MedEdPORTAL J Teach Learn Resour [Internet]. 2019 Jan 30 [cited 2019 Jul 18];15:10801. Available from: http://www.ncbi.nlm.nih.gov/pubmed/31044155

25. Dalcól C, Garanhani ML, Fonseca LF, Carvalho BG. Competência em comunicação e estratégias de ensino-aprendizagem: percepção dos estudantes de enfermagem. Cogitare Enferm [Internet]. 2018 Aug 8 [cited 2019 Jul 29];23(3). Available from: https://revistas.ufpr.br/cogitare/article/view/53743

26. Reuter CLO, Santos VCF dos, Ramos AR, Reuter CLO, Santos VCF dos, Ramos AR. The exercise of interprofessionality and intersetoriality as an art of caring: innovations and challenges. Esc Anna Nery [Internet]. 2018 Oct 29 [cited 2019 Jul 29];22(4).

Available

from:http://www.scielo.br/scielo.php?script=sci_arttext\&pid=S14148145201800040022 $1 \& \operatorname{lng}=$ en\&tlng=en

27. Villar F, Serrat R. Hable con ellos: cuidados narrativos en el marco de una atención centrada en la persona. Rev Esp Geriatr Gerontol [Internet]. 2017 Jul 1 [cited 2019 Jul 20];52(4):216-22. Available from: https://linkinghub.elsevier.com/retrieve/pii/S0211139X16300762

28. Siles González J. La humanización del cuidado a través de las narrativas y la poesía como producto de la investigación aplicada. Cult los Cuid Rev Enfermería y Humanidades [Internet]. 2018 [cited 2019 Jul 18];(52). Available from: 
http://hdl.handle.net/10045/85348

29. Ceballos Ramos LM, Rodríguez Flores LA, González Corrales SC, Ceballos Ramos LM, Rodríguez Flores LA, González Corrales SC. La metodología de la investigación cualitativa como necesidad en la carrera de licenciatura en enfermería. Mendive Rev Educ [Internet]. 2018 [cited 2019 Jul 30];16(3):470-83. Available from: http://scielo.sld.cu/scielo.php?script=sci_arttext\&pid=S1815-76962018000300470 30. Guillaumet M, Amorós G, Ramos A, Campillo B, Martínez Momblan MA. La narrativa como estrategia didáctica para una aproximación al proceso de la muerte. Enfermería Glob [Internet]. 2017 Dec 30 [cited 2019 Jul 18];17(1):185. Available from: http://revistas.um.es/eglobal/article/view/260491 